\title{
Inhibition of signal transduction pathways involved in inflammation
}

\author{
G. Haegeman
}

In order to target inflammatory processes effectively, it is necessary to understand, within an eukaryotic cell, how inflammatory reactions are generated and maintained. Therefore, this article will first concentrate on the signal transduction pathways and molecular mechanisms that drive inflammatory gene expression, followed by countering some of the published hypotheses that try to explain the inhibitory role of glucocorticoids. The current working model is included, which explains glucocorticoid action, and additionally points to an alternative or cooperative way of how to block inflammatory gene expression.

\section{Introduction}

Although inflammatory processes produce different diseases, depending on the inflamed tissue or organ involved, all of these afflictions have common aspects or common cellular processes, such as the activation of a stress signalling pathway and the concomitant production of inflammatory cytokines. In order to understand the inflammatory process at the "molecular" level and thus devise more specific molecular targets for the development of novel anti-inflammatory drugs, the author's group has studied signal transduction pathways and gene regulatory mechanisms that drive the expression of inflammatory genes. In particular, they studied the induction of interleukin (IL)-6 and other related gene promoters in mouse fibroblasts, in response to inflammatory stimuli such as tumour necrosis factor (TNF), and found that the transcription factor nuclear factor (NF)- $\kappa \mathrm{B}$ is crucial for transcriptional induction of these genes. This factor is a heterodimeric complex, composed of two subunits, a p50 deoxyribonucleic acid (DNA)-binding subunit and a p65 DNA-binding and transcriptionally active subunit. In the resting state of the cell, the NF- $\kappa \mathrm{B}$ complex is stored and kept inactive in the cell by an inhibitory molecule, I $\kappa$. As a result of various inflammatory stimuli, this inhibitor becomes subject to complete targeted degradation, releasing the $\mathrm{NF}-\kappa \mathrm{B}$ complex, allowing it to migrate to the nucleus and to bind onto NF- $\mathrm{NB}-$ responsive sequences present in various cellular promoters. This may lead to the recruitment of necessary cofactors and will give rise, together with the basal transcription machinery, to the induction of NF- $\kappa \mathrm{B}$-driven genes. Furthermore, the I $\kappa \mathrm{B}-\alpha$ gene (a subtype of the class of $\mathrm{I} \kappa \mathrm{B}$ inhibitors and clearly targeted in the case of inflammatory stimulation) also contains an NF- $\mathrm{B}$-responsive element in its promoter sequence, which leads to upregulation by $\mathrm{NF}-\kappa \mathrm{B}$ and to resynthesis of the inhibitor molecule that can now catch and

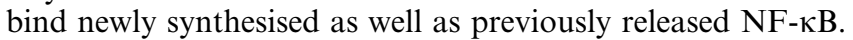
This acts as an autoregulatory loop (negative feedback) mechanism, keeping the NF- $\mathrm{B}$ levels in balance and gene transcription under tight control.

Correspondence: G. Haegeman, Dept of Molecular Biology, University of Gent, K.L. Ledeganckstraat 35, BE-9000, Gent, Belgium. Fax: 32 92645304. E-mail: guy.haegeman@UGent.be

\section{Dual activation pathways for inflammatory genes}

Inflammatory gene promoters are complex promoter sequences, comprising many different responsive sequence elements; however, it was found that within this multitude of transcription factor-binding possibilities, the transcription factor $\mathrm{NF}-\kappa \mathrm{B}$ (only and exclusively) is responsible for gene induction in response to TNF. Other promoter-bound factors (like activator protein-1, cyclic adenosine monophosphate responsive element-binding protein, CCAAT enhancerbinding protein, etc.) synergise with $\mathrm{NF}-\kappa \mathrm{B}$ and together form a necessary platform for recruitment of additional nuclear, non-DNA-bound cofactors (co-activators), which determine the general "levels" of inflammatory gene expression, yet not the induction of it. Taken together, the inducible factor $N F-\kappa B$ can be regarded as the ultimate switch for inflammatory gene induction, but, at the promoter level, it acts in concert with neighbouring promoter-bound transcription factors and associated, non-DNA-bound nuclear coactivators in order to drive inflammatory gene expression. The entire associated structure of promoter-defined and thus DNA-bound transcription factors, together with the specifically recruited, non-DNA-bound nuclear cofactors is called an "enhanceosome" and this stucture is found to be an essential step towards gene transcription [1].

Pretreatment of TNF-stimulated cells with inhibitors of the extracellular signal-related kinase (ERK) and/or the p38 mitogen-activated protein kinase (MAPK) pathways extinguishes inflammatory gene expression, but has no impact on cytoplasmic NF- $\kappa \mathrm{B}$ activation, nor on NF- $\kappa \mathrm{B} / \mathrm{DNA}$ binding. Therefore, it was hypothesised that, instead of the activation of $\mathrm{NF}-\kappa \mathrm{B}$, the transactivation capacity of the bound complex (namely the p65 subunit) may be disturbed by these types of inhibitors, i.e. by blocking phosphorylation cascades [2]. Neither ERK kinase nor p38 MAPK were shown to directly phosphorylate the NF- $\mathrm{B}$ p 65 subunit; the mitogen- and stress-activated protein kinase (MSK) 1 was found to be the executing enzyme for p65 phosphorylation. In fact, this kinase, which is downstream of ERK and of p38 in both these MAPK pathways, is a pure nuclear kinase that integrates upstream incoming signals for cell growth or stress. Blocking this particular kinase therefore equals inhibition of inflammatory gene expression.

The author's group also has clear evidence that MSK1 associates with NF- $\mathrm{B}$ p65 and, moreover, that the MSK1/ p65 association takes place at the level of the IL-6 promoter. However, this only occured when the cells were stimulated with TNF, and not in the resting state nor when the MAPK pathways or when MSK1 were blocked; this is to say that MSK1/p65 interaction and consequent phosphorylation is a specific, inflammatory stimulus-dependent event. MSK1 phosphorylates a specific residue (i.e. Ser 276) within the NF$\mathrm{\kappa B}$ p65 subunit. This amino acid position is a very critical residue, as mutation to another amino acid completely abolishes the transcriptional potency of NF- $\mathrm{B}$ p65. Indeed, 
p65 molecules, with a mutation at this particular site, are no longer able to contribute to the formation of the transcriptional-competent enhanceosome, and consequently unable to drive gene expresssion.

MSK1 is not a novel kinase, but was described to specifically phosphorylate the protruding peptide tails of the histon proteins within the nucleosomal structure. Modification of these tails is presently being considered as a "local coding" process within the chromatin to define its further tasks. In this hypothesis, chromatin modification leading to relaxation is parallelled with enhanced gene expression. Therefore, MSK1 seems to serve a double function, i.e. as a specific NF-кB p65 kinase, as well as an important signalling intermediate for chromatin relaxation [3].

In summary, the present model of inflammatory gene induction involves a dual signalling pathway (fig. 1). On the one hand, the transcription factor $N F-\kappa B$ is released from its inhibitory molecule and is able to bind onto various inflammatory gene promoters. On the other hand, the MAPK pathways are concomitantly activated and will lead to specific phosphorylation of the NF- $\mathrm{B}$ p65 subunit by MSK1 at a crucial amino acid residue, which is essential for enhanceosome building, while MSK1, having been recruited at inflammatory promoter regions, also phosphorylates the surrounding chromatin tails, contributing to local chromatin relaxation and facilitating gene transcription.

\section{Gene repression by glucocorticoids}

Glucocorticoids are known as effective drugs to combat inflammatory conditions, and are very widely used for this purpose. These lipophilic compounds can penetrate through the cellular membrane and bind to a specific acceptor molecule, the glucocorticoid receptor (GR). The receptor is released from a huge, cytoplasmic protein complex and migrates as a liganded carrier into the cell nucleus where it adopts its position as an activated transcription factor. It will thus bind onto so-called (mainly palindromic) glucocorticoid-responsive sequence elements within various gene promoters and collaborate with other factors to establish an enhanceosomic structure, necessary for gene expression. The anti-inflammatory potential of glucocorticoids, however, has not been associated with the gene-inductive activities of the activated GR, but rather assumed to result from "negative interference" with inflammatory, i.e. NF- $\mathrm{BB}-d$ riven gene expression. A few authors have pointed to the potential of glucocorticoids, i.e. of the activated GR, to upregulate expression of the gene coding for the NF- $\kappa \mathrm{B}$-inhibitor molecule I $\kappa \mathrm{B}-\alpha[4,5]$, but the present author's group has not found this regulatory effect of GR in fibroblast or endothelial cells, nor did they observe diminished NF- $\kappa \mathrm{B}$ binding with glucocorticoid treatment. On the contrary, they have demonstrated that glucocorticoid inhibition of $\mathrm{NF}-\kappa \mathrm{B}$ activity takes place in the absence of gene transcription and protein synthesis, and occurs entirely in the cell nucleus [6]. Other investigators considered nuclear GR as a competitor for activated $\mathrm{NF}-\kappa \mathrm{B}$ in assembling the necessary enhanceosome at gene promoters, resulting in firmly decreased NF- $\kappa \mathrm{B}$ gene transcription [7, 8]. The author's group found that this is not the case, and established that NF- $\mathrm{KB}$ remains in contact with enhanceosomic partners even in conditions of repression by GR. Moreover, this hypothesis proposes a general way of gene repression and does not explain the specificity of glucocorticoids towards inflammation $[9,10]$. No effect of glucocorticoids on chromatin modification, nor any interference with the abovementioned phosphorylation cascades was found. This is to say that the necessary "MAPK $\rightarrow$ MSK $1 \rightarrow$ p65 transactivation"

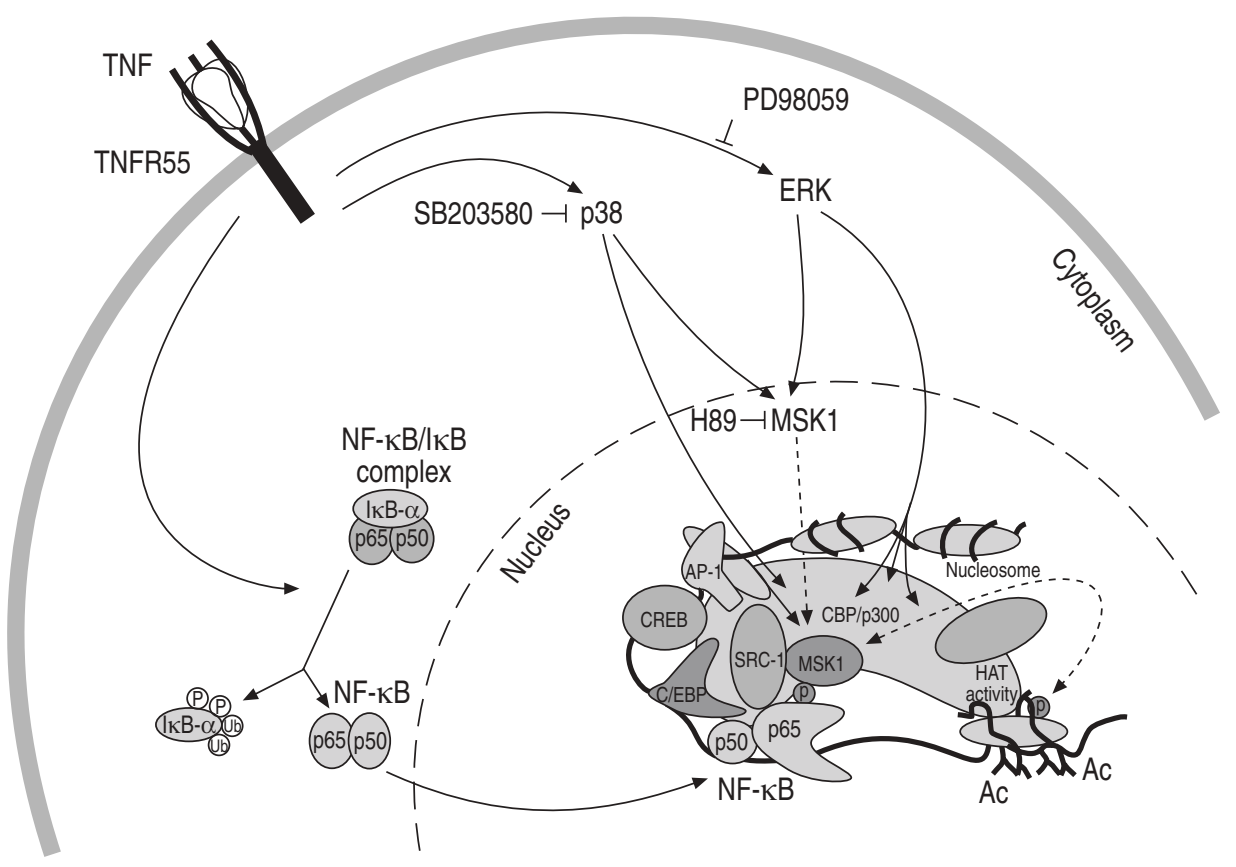

Fig. 1.-Tumour necrosis factor (TNF) activates inflammatory gene expression via a dual signalling pathway. The figure shows that upon triggering of the membrane-bound TNF receptor R55, the cytoplasmic activation of the transcription factor complex nuclear factor (NF)- $\mathrm{B}$ transfers to the nucleus and takes its position at the NF- $\kappa$ B-responsive element in the interleukin-6 promoter next to other already constitutively bound transcription factors. Concomitantly, the mitogen-activated protein kinases, p38 and extracellular signal-related kinase (ERK), are also activated by TNF induction, followed by activation of their common nuclear substrate, mitogen- and stress-activated protein kinase (MSK)1, which phosphorylates NF- $\mathrm{NB}$ p65 at a crucial residue to make it a suitable interaction partner for cyclic adenosine monophosphate-responsive element-binding protein (CREB)-binding protein (CBP)/p300 and other nuclear coactivators. Histon tails become phosphorylated by MSK1, and acetylated by nuclear coactivators in order to relax the chromatin and enhance gene expression. AP: activator protein; SRC: steroid receptor coactivator; C/EBP: CCAAT enhancer-binding protein; p/CAF: p300/CBP activating factor; HAT: histon acetyl transferation; Ac: acetyl. 


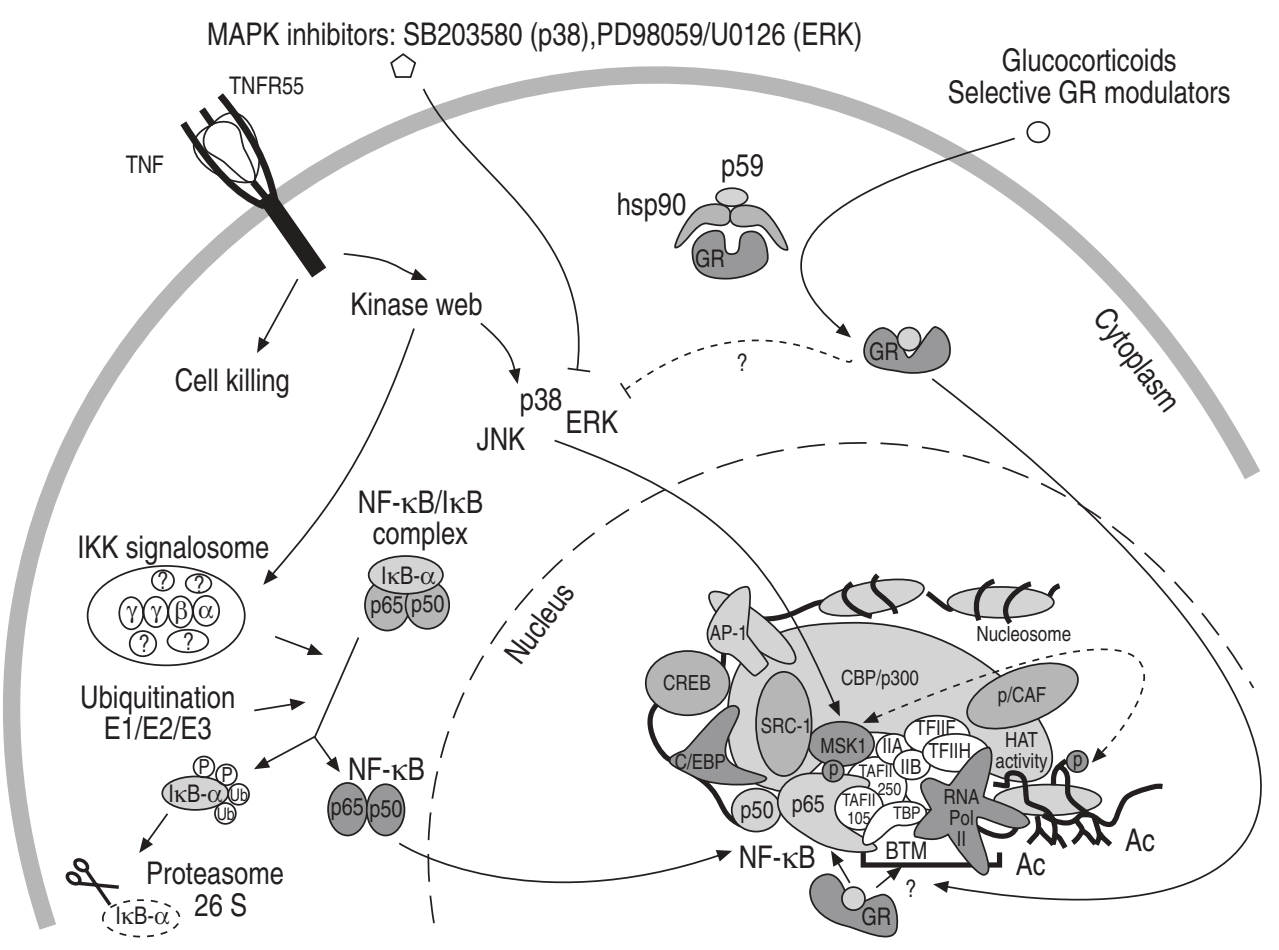

Fig. 2.-Glucocorticoids bind to the glucocorticoid receptor (GR), which translocates into the nucleus and inhibits inflammatory gene expression in a promoter architecture-specific way. This figure shows the same tumour necrosis factor (TNF)-driven gene activation pathways as shown in figure 1. However, upon addition of glucocorticoids, the GR is released from a cytoplasmic protein complex and transferred to the nucleus, where, in case of gene repression, it specifically interacts with the activating interface between nuclear factor (NF)- $\mathrm{kB}$ p65 and the basal transcription machinery (BTM), thus inhibiting gene expression in a promoter-specific way. Note that GR does probably not interfere with the mitogen-activated protein kinase (MAPK) cascade. AP: activator protein; CREB: cyclic adenosine monophosphate-responsive element-binding protein; C/EBP: CCAAT enhancer-binding protein; ERK: extracellular signal-related kinase; JNK: Jun N-terminal kinase; RNA: ribonucleic acid; SRC: steroid receptor coactivator; p/CAF: p300/CREB-binding protein (CBP) activating factor; HAT: histon acetyl transferation; Ac: acetyl; TBP: TATA-binding protein.

pathway is not affected by glucocorticoids and may thus contain alternative targets for an anti-inflammatory strategy, either or not in combination with glucocorticoid treatment.

The author's present working model proposes GR to specifically disturb the interaction platform between $N F-\kappa B$ p65 and the basal transcription machinery, in which specificity of gene repression is guaranteed by the setting and the spatial organisation of the various factors involved, such as that dictated by the primary sequence of the inflammatory gene promoters.

\section{Conclusion}

To conclude, the occurrence and necessity of a dual signalling pathway for the transcriptional activation of nuclear factor- $\kappa \mathrm{B}-$ driven inflammatory genes has been demonstrated, in which specificity for inflammatory gene promoters may somehow be regulated or dictated by concomitant chromatin relaxation. Upon inflammatory gene repression by glucocorticoids, the transcriptional-competent complexes and chromatin structure remain unaffected; however, activated glucocorticoid receptor seems to interfere with the contacts between nuclear factor- $\kappa \mathrm{B}$ p65 and the basal transcription complex, and thus downmodulates gene expression in a promoter architecture-specific way [11] (fig. 2).

\section{References}

1. Vanden Berghe W, De Bosscher K, Boone E, Plaisance S, Haegeman G. The nuclear factor- $\mathrm{KB}$ engages $\mathrm{CBP} / \mathrm{p} 300$ and histone acetyltransferase activity for transcriptional activation of the interleukin-6 gene promoter. J Biol Chem 1999; 274: 32091-32098.

2. Vanden Berghe W, Plaisance S, Boone E, et al. p38 and extracellular signal-regulated kinase mitogen-activated protein kinase pathways are required for nuclear factor- $\kappa \mathrm{B}$ p65 transactivation mediated by tumor necrosis factor. $J$ Biol Chem 1998; 273: 3285-3290.

3. Vermeulen L, De Wilde G, van Damme P, Vanden Berghe W, Haegeman G. Transcriptional activation of the NF- $\kappa B$ p65 subunit by mitogen- and stress-activated protein kinase1 (MSK1). EMBO J 2003; 22: 1-12.

4. Scheinman R, Cogswell P, Lofquist A, Baldwin A. Role of transcriptional activation of $\mathrm{I}-\kappa \mathrm{B}-\alpha$ in mediation of immunosuppresssion by glucocorticoids. Science 1995; 270: 283286.

5. Auphan N, DiDonato J, Rosette C, Helmberg A, Karin M. Immunosuppression by glucocorticoids: inhibition of NF- $\kappa \mathrm{B}$ activity through induction of I-кB synthesis. Science 1995; 270: 286-290.

6. De Bosscher K, Schmitz ML, Vanden Berghe W, Plaisance S, Fiers W, Haegeman G. Glucocorticoid-mediated repression of nuclear factor- $\kappa \mathrm{B}$-dependent transcription involves direct interference with transactivation. Proc Natl Acad Sci USA 1997; 94: 13504-13509.

7. Kamei Y, Xu L, Heinzel T, et al. A CBP integrator complex mediates transcriptional activation and AP-1 inhibition by nuclear receptors. Cell 1996; 85: 403-414.

8. Sheppard K, Phelps K, Williams A, et al. Nuclear integration of glucocorticoid receptor and nuclear factor- $\kappa \mathrm{B}$ signaling by CREB-binding protein and steroid receptor coactivator-1. J Biol Chem 1998; 273: 29291-29294.

9. De Bosscher K, Vanden Berghe W, Vermeulen L, 
Plaisance S, Boone E, Haegeman G. Glucocorticoids repress $\mathrm{NF}-\kappa \mathrm{B}$-driven genes by disturbing the interaction of p65 with the basal transcription machinery, irrespective of coactivator levels in the cell. Proc Natl Acad Sci USA 2000; 97: 3919-3924.

10. De Bosscher K, Vanden Berghe W, Haegeman G. Glucocorticoid repression of AP-1 is not mediated by competition for nuclear coactivators. Mol Endocrinol 2001; 15: 219227.

11. De Bosscher K, Vanden Berghe W, Haegeman G. Mechanisms of anti-inflammatory action and of immunosuppression by glucocorticoids: Negative interference of activated glucocorticoid receptor with transcription factors. J Neuroimmunol 2000; 109: 16-22. 